\title{
C-Quand et hôtes non verbaux : entre syntaxe et sémantique
}

\author{
Frédérique Saez ${ }^{*}$ \\ Université de Guyane, Route de Baduel, 97300 Cayenne
}

\begin{abstract}
Résumé. La présente communication se propose d'examiner un phénomène fréquent mais peu décrit du français contemporain. Il s'agit des cas où un constituant introduit par quand parait entrer dans la dépendance d'un constituant autre que le verbe. D'un point de vue sémantique, il parait délicat de douter du rapport entre cet hôte non canonique du C-quand et le $C$-quand, mais d'un point de vue syntaxique, les liens sont parfois très ténus, voire difficiles à montrer. Nous examinerons donc ces phénomènes d'un point de vue syntaxique et sémantique, espérant ainsi contribuer à une meilleure compréhension des phénomènes d'intégration des constructions verbales.
\end{abstract}

\begin{abstract}
C$-quand and host other than verbs: between syntax and semantic. The present communication suggests examining a phenomenon frequent but little described by contemporary French. It is cases where a constituent introduces by quand adorned to enter the dependence of a constituent other than the verb. From a semantic point of view, he countered delicate to doubt the report enter this not canonical host of $C$ quand and $C$-quand, but from a syntactic point of view, the links are sometimes very tenuous, even difficult to show. Thus we shall examine these phenomena by a syntactic and semantic examination, so hoping to contribute to a better understanding of the phenomena of integration of the verbal constructions.
\end{abstract}

\section{Introduction}

\subsection{Objectifs}

La présente étude se propose d'examiner une structure fréquente du français mais peu voire pas - décrite par les grammaires traditionnelles. Il s'agit des cas où un constituant ${ }^{\mathrm{i}}$ introduit par quand (désormais $C$-quand) semble se trouver dans la dépendance ${ }^{\mathrm{ii}}$ non canonique d'un élément ${ }^{\mathrm{iii}}$ autre que le verbe.

En effet, le $C$-quand peut se trouver dans la dépendance de constituants de type nominal pour lesquels l'attachement du $C$-quand au syntagme nominal (désormais $\mathrm{SN}$ ) parait difficilement contestable :

1. Elle riait. Cette odeur... C'était celle de Mathilde quand elle était petite...

(Frantext (désormais FT) : Gavalda A., La Consolante, 2008) ${ }^{\text {iv }}$

\footnotetext{
*Corresponding author : frederique.saez@ac-guyane.fr
} 
2. De qui a-t-il peur? De toi, quand tu sais et quand il croit être le seul à savoir ?

De toi quand tu souris, un sourire, un vrai, pas celui des courtoisies ou bien l'autre, blet, des jaloux? (FT : Navarre Y., Je vis où je m'attache, 1978)

En (1) et (2) le $C$-quand semble bien compléter (syntaxiquement et sémantiquement) le nom ou pronom mais pas le verbe qui précède. Toutefois, dans certains exemples on peut hésiter quant au rattachement au nom, ainsi dans (3) et (4) ci-dessous :

3. L'actuelle vague de violences s'est ouverte fin novembre quand, en l'espace d'une seule journée, plus de 40 personnes ont été tuées à Amboise. (Journal Le Monde (désormais JLM), 12/99)

4. Les heures de la contestation, quand Beaubourg était moqué pour ses allures d'usine à gaz, semblent bien lointaines ! (JLM, 12/99)

Ici deux interprétations semblent a priori possibles: analyser le $C$-quand comme dépendant du verbe, entrant alors dans le même paradigme que le SN antécédent, soit analyser le $C$-quand comme dépendant de ce $\mathrm{SN}^{\mathrm{V}}$.

On trouve la même difficulté d'identification de la cible du $C$-quand dans les exemples où il parait lié à un syntagme adjectival (désormais SAdj.) :

5. Mais elle n'a pas peur du tout, elle a appris à lancer ses billes d'un bref coup de pouce et son regard sait être insolent quand il faut. (FT : Fellous C., Avenue de France, 2001)

En revanche son attachement à l'adverbe (6) ou à la préposition (7) semble moins contestable :

6. « et maintenant, quand les seigneurs se battent, c'est nous les pauvres qui souffrons... » (JLM, 12/99)

7. Je ne vois jamais aucune autorité publique se manifester dans les ruelles, ni aucun uniforme se profiler sur la place, sauf quand le facteur vient de Sermano distribuer le courrier. (FT : ARNAUD C., Qu'as-tu fait de tes frères ?, 2010)

Dans certains emplois, il semblerait de plus que l'ensemble \{hôte $+C$-quand $\}$ tende à une certaine autonomie énonciative :

8. ça y est ! dit La Menace, un zig qui veut de l'essence! Faut que j'y aille! Bon sang, toujours quand il faut pas! (FT : Thérame V., Bastienne, 1985)

Nous nous proposons donc d'analyser les différentes occurrences où $C$-quand semble dans la dépendance d'un constituant autre que le verbe, en examinant dans le détail la syntaxe de ces $C$-quand pour tenter de circonscrire la nature de la relation entre l'hôte et le $C$-quand, puis - le cas échéant - la nature de la relation entre le couple \{hôte $+C$-quand $\}$ et le reste de l'énoncé. Ceci nous conduira à revenir sur le statut de quand.

Le corpus de travail utilisé sera essentiellement issu de la base textuelle Frantext, d'internet, ainsi que de nos lectures et travaux personnels.

\subsection{Cadre descriptif}

Au plan syntaxique, nous empruntons à l'Approche pronominale telle que définie et développée par Van den Eynde et al. (1978) et Blanche-Benveniste et al. (1984), en nous appuyant essentiellement sur les travaux subséquents de Smesseart et al. (2005). Pour distinguer les niveaux d'intégration syntaxique, les auteurs utilisent les manipulations suivantes :

- la proportionnalité pronominale (proportionality), ou la possibilité pour un constituant de commuter avec un pronom suspensif (9a) ou assertif (9b) ;

- l'extraction d'un constituant (clefting) avec ou sans reprise pronominale (9c); 
- et l'ordre séquentiel (désormais OS) de l'énoncé, avec ou sans reprise pronominale (fronting and left-dislocation) (9c).

Par exemple voici les résultats pour un $C$-quand en position canonique de circonstant :

9. J'aimerais sympathiser avec eux et leur dire que j'ai appris l'hébreu quand j'étais petite à l'école Maïmonide et que si je n'en connais pas davantage c'est la faute de mon père. (FT : Akerman Ch., Ma mère rit, 2013)

a. proportionnalité avec un pronom suspensif : j'ai appris l'hébreu quand ? Quand j'étais petite.

b. proportionnalité avec un pronom assertif : j'ai appris l'hébreu à ce moment-

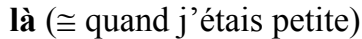

c. extraction: C'est (à ce moment-là) quand j'étais petite que j'ai appris l'hébreu.

d. OS de l'énoncé : Quand j'étais petite, j'ai appris l'hébreu (à ce moment-là).

L'utilisation de ces manipulations permet de mettre en évidence les contraintes qui pèsent sur les structures et donc de circonscrire la nature de la relation entre le constituant hôte et le $C$-quand. Nous associerons à cette analyse de la syntaxe externe, un examen de la syntaxe interne et des contraintes sémantiques qui sont susceptibles de peser sur le $C$ quand.

\section{Propriétés syntaxiques et sémantiques des occurrences}

Afin de ne pas présupposer ou anticiper sur la nature de la relation entre le $C$-quand et le constituant hôte, les occurrences seront classifiées selon la catégorie syntaxique de ce dernier.

\subsection{Structure $\{\mathrm{SN}+\mathrm{C}$-quand $\}$}

Dans cette section nous examinerons les cas où le $C$-quand se trouve attaché à un $\mathrm{SN}$, qui entretient avec $C$-quand une relation sémantique et temporelle particulière. Nous distinguerons les occurrences selon le sémantisme du SN antécédent.

Lorsque le $C$-quand a pour antécédent un $\mathrm{SN}$ animé (désormais $\mathrm{SN}_{/ \text {animél}}$ ) du type de (1) et (2) ci-dessus, il est aisé de montrer la solidarité entre les deux constituants en raison de ce qu'il est impossible d'extraire le $C$-quand seul (10a), de lui substituer seul un pronom assertif ou interrogatif (10b) ou encore de l'antéposer seul à l'énoncé (10c) contrairement au $C$-quand canonique cité supra (9) :

10. Cette odeur... C'était celle de Mathilde quand elle était petite... (déjà cité 1)

a. ? C'est quand elle était petite que c'était l'odeur de Mathilde.

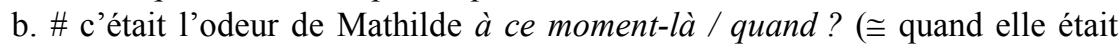
petite).

c. ? quand elle était petite, c'était l'odeur de Mathilde.

Alors que l'extraction de l'ensemble $\left\{\mathrm{SN}_{\text {/animél }}+C\right.$-quand $\}$ ne pose pas de problème :

11. De qui a-t-il peur? De toi quand tu souris (déjà cité 2)

a. C'est de toi quand tu souris qu'il a peur.

ni même la proportionnalité de l'ensemble avec un indéfini :

12. Tu ferais mieux de rester tranquille. Et puis de moins t'en faire. Tu t'en fais trop. Mais non. Mais si, je le vois bien. Toi aussi tu t'en fais. Oui, parfois mais pas comme toi. \{Toi quand tu t'en fais\} cela devient vite grave et dangereux pour toi. (FT : Akerman Ch., Ma mère rit, 2013) 
Dans le cadre de l'Approche Pronominale, Benzitoun (2006: 130 et suiv.) analyse ces structures $\left\{\mathrm{SN}_{/ \text {animé/ }}+C\right.$-quand $\}$ comme des nexus. À l'appui de cette hypothèse vient la proportionnalité de l'ensemble avec la proforme ça/cela (cf. 12 supra) ou encore la syntaxe interne relativement contrainte, entre autre par la coréférentialité obligatoire entre le SN antécédent et le sujet du $C$-quand, faute de modifier la structuration :

13. - Tu es courageuse, dit Mama Can, tout moi quand j'avais ton âge, le travail ne m'a jamais fait peur. (FT : Levy M., L'étrange voyage de Monsieur Daldry, 2011)

a. ...tout moi quand il pleut.

En (13a) le $C$-quand redevient un régi ordinaire.

Nous rejoindrons l'analyse de l'auteur pour ces structures.

En revanche, dans les cas où le $C$-quand parait dans la dépendance d'un SN à valeur temporelle (désormais $\mathrm{SN}_{\text {/temps/) }}$ ) le $C$-quand s'apparenterait à une relative ${ }^{\mathrm{vi}}$. Dans ces cas-là nous distinguerons entre deux types de $\mathrm{SN}$ : les $\mathrm{SN}$ exprimant une datation (désormais

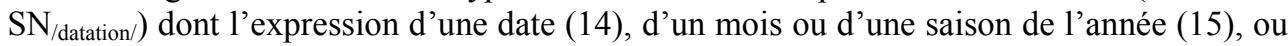
encore d'un jour de la semaine (16) :

14. Une légende entoure ce réfugié apatride dont la famille a fui l'Egypte en 1956, quand Nasser expulsa les Juifs et les étrangers. (FT : Arnaud Cl., Qu'as-tu fait de tes frères?,2010)

15. L'hiver, quand le jour tarde à poindre, ce sont des morts-vivants qui semblent sortir de leur tombe en tenue de deuil. (FT : Arnaud Cl., Qu'as-tu fait de tes frères?,2010)

16. À l'angle se niche un café sans nom - on dit qu'on va «chez le bougnat »-, un café de quartier où les hommes viennent jouer à la belote le samedi quand la mère a besoin de place pour faire la toilette des enfants. (FT : Jablonka I., Histoire des grands-parents que je n'ai pas eus : une enquête, 2012)

et les $\mathrm{SN}$ exprimant une période historique (désormais $\mathrm{SN}_{\text {/période/ }}$ ) :

17. Les heures de la contestation, quand Beaubourg était moqué pour ses allures d'usine à gaz, semblent bien lointaines ! (JLM 12/99)

18. Comme la pernicieuse habitude de la confession, imposée dès l'enfance, quand il faut se torturer pour trouver des peccadilles à chuchoter vers le grillage qui les attend. (FT : de Margerie D., Le Ressouvenir, 1985)

Pour ces occurrences il est aisé de montrer que le $\mathrm{SN}_{\text {/temps/ }}$ et le $C$-quand entretiennent une relation syntaxique particulière, puisqu'il est impossible d'extraire le $C$-quand seul (19a et 20a), de lui substituer seul un pronom assertif ou interrogatif (19b et 20b) ou encore de l'antéposer seul à l'énoncé (19c et 20c) contrairement au C-quand canonique cité supra (9) :

19. L'actuelle vague de violences s'est ouverte fin novembre quand, en l'espace d'une seule journée, plus de 40 personnes ont été tuées à Amboise. (JLM, 12/99)

a. ? C'est quand plus de 40 personnes ont été tuées à Amboise que l'actuelle vague de violences s'est ouverte fin novembre.

b. ? L'actuelle vague de violences s'est ouverte fin novembre à ce moment-là /

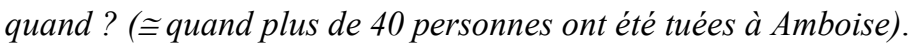

c. ? quand plus de 40 personnes ont été tuées à Amboise l'actuelle vague de violences s'est ouverte fin novembre.

20. elle est tellement incapable de penser, les jours de départ, quand il faut se quitter. (FT : Cluny Cl.-M., Un jeune homme de Venise, 1983) 
a. ? C'est quand il faut se quitter qu'elle est incapable de penser les jours de départ.

b. ? elle est tellement incapable de penser, les jours de départ, à ce moment-là /

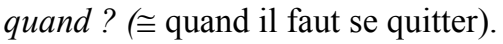

c. ? quand il faut se quitter elle est incapable de penser les jours de départ.

Alors que l'extraction de l'ensemble $\left\{\mathrm{SN}_{/ \text {temps/ }}+C\right.$-quand $\}$ ne pose pas de problème (19d et 20d) ni même la proportionnalité de l'ensemble avec un pronom assertif ou interrogatif (19e et 20e) :

19 d. C'est fin novembre quand plus de 40 personnes ont été tuées à Amboise que l'actuelle vague de violences s'est ouverte.

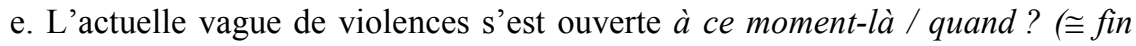
novembre quand plus de 40 personnes ont été tuées à Amboise).

20 d. C'est les jours de départ quand il faut se quitter qu'elle est incapable de penser.

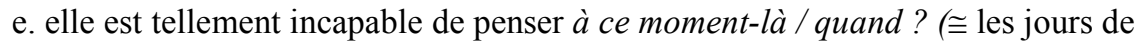
départ quand il faut se quitter).

Benzitoun (2006 : 276 et suiv.) analyse les $C$-quand hôte d'un $\mathrm{SN}_{/ \text {datation/ (soit } 14 \text { à } 16}$ supra) comme de simples ajouts en raison de ce que «la relation ne s'instaure pas entre l'élément temporel et la Quand-C mais entre la Quand-C et l'ensemble de la construction verbale qui la précède $»$. En effet dans ces clauses on peut reconnaitre l'imbrication de trois éléments temporels ${ }^{\mathrm{vii}}$ les uns dans les autres, du plus général au plus précis :

21. Il pourra partir lundi quand il aura fini ses devoirs (cité par Benzitoun, 2006 : 271)

a. [Il pourra partir + [datation : lundi]] $T \subset^{\text {viii }}$ [quand il aura fini ses devoirs].

Si la relation d'inclusion temporelle est indéniable, il n'en reste pas moins que ces $C$ quand ont des propriétés distributionnelles bien éloignées des ajouts classiques (cf. (9) supra), il nous parait donc délicat de les considérer comme tels.

En revanche, ce type de C-quand réagit exactement comme le ferait une relative introduite par où dans le sens où cette dernière ne peut être extraite seule dans la construction clivée (22a), mais avec son antécédent (22b), qu'elle n'est pas proportionnelle seule à un pronom assertif (22c), mais avec son antécédent (22d), et qu'elle ne peut être antéposée seule (22e) :

22. Voracieux est le lieu où fermentent nos idées noires. (FT: Jenni A., L'Art français de la guerre, 2011)
a. ? C'est où fermentent nos idées noires que Voracieux est le lieu
b. C'est le lieu où fermentent nos idées noires qu'est Voracieux
c. ? Voracieux est le lieu là
d. Voracieux est là
e. ? Où fermentent nos idées noires Voracieux est le lieu

Le tableau ci-dessous récapitule et confronte les propriétés des structures $\left\{\mathrm{SN}_{/ \text {datation/ }}+\right.$ $C$-quand $\}$ en regard d'une traditionnelle circonstancielle (ou C-quand ajout dans la terminologie de l'Approche Pronominale) ou d'une relative : 
Tableau 1. Propriétés comparées des structures.

\begin{tabular}{|c|c|c|c|c|}
\hline \multicolumn{2}{|c|}{$\begin{array}{ll}\text { Propriétés } & \text { Structures }\end{array}$} & $\begin{array}{l}\text { C-quand } \\
\text { ajout }\end{array}$ & $\underset{\text { C-quand }}{\mathrm{SN}_{/ \text {datation/ }}+}$ & C-Où \\
\hline \multirow{2}{*}{$\begin{array}{l}\text { Extraction dans } \\
\text { la clivée }\end{array}$} & $\begin{array}{l}C \text {-quand/où } \\
\text { seul }\end{array}$ & + & - & - \\
\hline & L'ensemble & $\mathrm{NC}$ & + & + \\
\hline \multirow{2}{*}{ Proportionnalité } & $\begin{array}{l}\text { C-quand/où } \\
\text { seul }\end{array}$ & + & - & - \\
\hline & L'ensemble & $\mathrm{NC}$ & + & + \\
\hline \multirow{2}{*}{ Mobilité } & $\begin{array}{l}C \text {-quand/où } \\
\text { seul }\end{array}$ & + & - & - \\
\hline & L'ensemble & $\mathrm{NC}$ & + & + \\
\hline
\end{tabular}

$\mathrm{Au}$ vu de ce tableau, il semblerait plus judicieux de considérer le $C$-quand comme une relative plutôt que comme un ajout. Cette analyse est corroborée par les manipulations cidessous qui, d'après Riegel et al. (2009 : 204 et suiv.), permettent d'identifier une relative appositive, à savoir, sa possible suppression (23a), sa proportionnalité avec une proposition coordonnée (23b), l'insertion possible d'une incidente (23c) ou la reprise par cela (23d) :

23. a. L'hiver $\boldsymbol{\varnothing}$ ce sont des morts-vivants qui semblent sortir de leur tombe en tenue de deuil. (déjà cité 15)

b. L'hiver ce sont des morts-vivants qui semblent sortir de leur tombe en tenue de deuil car le jour tarde à poindre.

c. L'hiver, quand par ailleurs/entre autres choses le jour tarde à poindre, ce sont des morts-vivants qui semblent sortir de leur tombe en tenue de deuil.

d. L'hiver, quand le jour tarde à poindre (cela est systématique), ce sont des morts-vivants qui semblent sortir de leur tombe en tenue de deuil.

L'ensemble de ces manipulations tendrait donc à montrer que ce type particulier de $C$ quand est bien apparenté aux relatives appositives.

Pour les $\mathrm{SN}_{\text {/période/ }}$ Benzitoun propose (2006 : 272 et suiv.) de voir que le $\mathrm{SN}_{\text {/période/ }}$ et le $C$-quand n'occupent qu'une seule place syntaxique, i.-e. de considérer une liste distributive avec ou sans coïncidence temporelle entre les deux éléments. Dans ce sens, $C$-quand est dès lors analysé par Benzitoun (ibid.) comme une spécification sémantique du premier ajout, et dans la mesure où ce premier ajout et $C$-quand forment une seule et même liste distributive, il considère $C$-quand comme rectionnel.

Il rapproche ces structures $\left\{\mathrm{SN}_{\text {/période/ }}+C\right.$-quand $\}$ des énoncés ci-dessous qui présentent une liste distributive, où le $C$-quand est précédé de c'est-à-dire ou d'une énumération en et...et..., sans coïncidence temporelle :

24. donc les parlers approchés ou interlangue ou langue approximative + c'est l'autre cas de figure c'est-à-dire quand il n'y a pas moyen de communication + (PUB-PSE-1 > Benzitoun 2006 : 272)

25. J'ai connu dans ma jeunesse et quand les études de cet ordre m'intéressaient, plusieurs individus aussi emprisonnés que lui dans l'atmosphère des spéculations abstraites. (Disc. $<$ Sandfeld $<$ Benzitoun, $2006: 273$ ) 
Toutefois cette analyse en liste paradigmatique nous parait parfois délicate. En effet, il faudrait pour (4) considérer alors que le $C$-quand occupe une place de sujet, or la manipulation (26a) ci-dessous parait contredire ce point de vue :

26. Les heures de la contestation, quand Beaubourg était moqué pour ses allures d'usine à gaz, semblent bien lointaines! (déjà cité 4)

a. ? quand Beaubourg était moqué pour ses allures d'usine à gaz semble bien lointain

alors qu'il est possible de substituer à la structure $\left\{\mathrm{SN}_{\text {période/ }}+C\right.$-quand $\}$ un $\mathrm{SN}$ à valeur temporelle :

27. Ce moment-là semble bien lointain.

(27) montre la solidarité entre l'hôte et le $C$-quand, solidarité qui vient infirmer l'analyse en liste paradigmatique.

De plus, s'il s'agissait d'une liste, il serait possible de substituer au C-quand une proforme à valeur temporelle, or il semble que cette manipulation soit délicate :

28. ? Les heures de la contestation, ces moments-là, semblent bien lointains.

Lors de précédents travaux ${ }^{\text {ix }}$, nous avons également travaillé sur ces constructions $\left\{\mathrm{SN}_{/ \text {datation-période/ }}+C\right.$-quand $\}$ et valider l'hypothèse d'une distinction entre les $\mathrm{SN}_{/ \text {datation/ }}$ ou

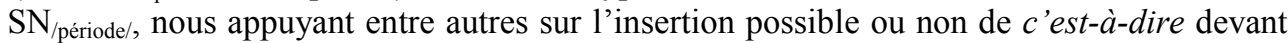
le C-quand (Saez, 2011 : 264 et suiv.) :

29. \# Une légende entoure ce réfugié apatride dont la famille a fui l'Egypte en 1956, c'est-à-dire quand Nasser expulsa les Juifs et les étrangers. (déjà cité 14)

30. Les heures de la contestation, c'est-à-dire quand Beaubourg était moqué pour ses allures d'usine à gaz, semble bien lointaines. (déjà cité 17)

Au plan sémantique, ce test d'insertion de c'est-à-dire montre le type de relation entre le $\mathrm{SN}_{/ \text {temps/ }}$ et $C$-quand. En effet, en présence d'un $\mathrm{SN}_{\text {/datation/ }}$ il s'agirait effectivement d'une inclusion temporelle, paraphrasable ${ }^{\mathrm{x}}$ par à un moment de $S N$ où :

31. Nous en sommes à tester la mescaline, début juillet 71, quand surgit un ami de Serge, sapé comme les minets de l'avant 68. (FT: Arnaud Cl., Qu'as-tu fait de tes frères?, 2010)

a. Nous en sommes à tester la mescaline, à un moment de début juillet 71 où surgit un ami de Serge, sapé comme les minets de l'avant 68.

En revanche, en présence d'un $\mathrm{SN}_{\text {/période/ }}$ il s'agirait plutôt d'une explicitation avec coïncidence temporelle.

En effet, il semblerait que ces structures fonctionneraient sémantiquement comme les occurrences suivantes :

32. Le soir tombait quand mon père rentra enfin. (Goosse-Grévisse, cité par Beguelin, $2004: 6$ )

Dans Saez (2011: 186 et suiv.) nous avions analysé le C-quand de (32) comme épitaxique (i.e. non comme un ajout classique, mais comme un constituant sémantiquement lié à ce qui précède (en l'occurrence le soir tombait), mais syntaxiquement détaché du verbe qui précède). Ce dégroupage du C-quand s'accompagne d'un certain nombre de contraintes, entre autres sur le temps et l'aspect du verbe de la section hôte, et sur la polarité du $C$-quand nécessairement positive. Ces contraintes s'expliquent aisément par la relation temporelle de recouvrement partiel ${ }^{\mathrm{xi}}$ entre les deux segments, ainsi que par le fait que le segment hôte est informationnellement peu saillant par rapport au $C$-quand.

Il semblerait que les choses soient équivalentes (d'un point de vue sémantique) dans les structures $\left\{\mathrm{SN}_{/ \text {période/ }}+C\right.$-quand $\}$, en atteste d'ailleurs la même contrainte de polarité positive sur le $C$-quand: 
33. ? Les heures de la contestation, quand Beaubourg n'était pas moqué pour ses allures d'usine à gaz, semblent bien lointaines. (déjà cité 17)

34. ? Comme la pernicieuse habitude de la confession, imposée dès l'enfance, quand il ne faut pas se torturer pour trouver des peccadilles à chuchoter [...] (déjà cité 18)

35. ? elle est tellement incapable de penser, les jours de départ, quand il ne faut pas se quitter. (déjà cité 20)

On remarque également que le $\mathrm{SN}_{\text {/période/ }}$ n'est informationnellement que peu saillant : les heures de la contestation (17), l'enfance (18), ou encore les jours de départ (20). L'actualisation de ces $\mathrm{SN}_{\text {/période/ }}$ par le défini générique comportent un sémantisme trop vaste pour qu'il puisse être ancré. De fait, on peut donc considérer que ces $\mathrm{SN}_{\text {/période/ }}$ préparent d'une certaine façon le contenu informationnel du $C$-quand qui se présente dès lors comme une sorte de parenthèse explicative, sur laquelle est mise la focale du locuteur. Le $\mathrm{SN}_{\text {/période/ }}$ offre donc un point d'ancrage au C-quand qui porte l'information pertinente.

Mais on remarque qu'il en va de même lorsque le $\mathrm{SN}_{\text {/période/ }}$ est plus spécifié, dans nos exemples respectivement par un déterminant possessif (36) ou par un syntagme prépositionnel (37) :

36. Nana croyait retourner à ses débuts, quand sa première robe de soie lui avait causé un si gros plaisir. (Zola $<$ TLFi)

37. il a monté nos escaliers, il a défoncé nos portes et ça a été le commencement de la misère, le commencement du manque d'argent, le commencement de l'obscurité quand il faut de la lumière et des soleils qui refusent de se coucher. (FT : Koltès B.-M., Quai ouest, 1985)

Dans ce cas on observe une nouvelle contrainte sur le $C$-quand, il doit y avoir une relation lexicale entre le $\mathrm{SN}_{\text {/période/ }}$ et le $C$-quand. Pour (36) cette relation est assurée par la présence des déterminants possessifs : ses et $s a$, qui co-actualisent respectivement les SN débuts et première robe de soie. Alors que dans (37) cette cohérence est assurée par la présence d'antonymes, suivis d'une isotopie antonymique : obscurité vs. lumière/soleil.

En (36) et (37) toutefois, si le $C$-quand n'est indéniablement pas régi par le verbe qui le précède comme en attestent les manipulations suivantes :

38. a. extraction :

? c'est quand sa première robe de soie lui avait causé un si gros plaisir que Nana croyait retourner à ses débuts.

? c'est quand il faut de la lumière et des soleils qui refusent de se coucher que ça a été le commencement de l'obscurité.

b. proportionnalité avec un pronom assertif ou interrogatif :

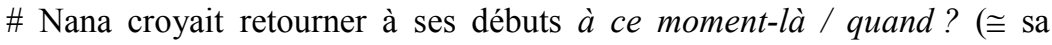
première robe de soie lui avait causé un si gros plaisir).

\# ça a été le commencement de l'obscurité à ce moment-là / quand ? ( quand il faut de la lumière et des soleils [...]).

c. mobilité :

? quand sa première robe de soie lui avait causé un si gros plaisir, Nana croyait retourné à ses débuts.

? quand il faut de la lumière et des soleils qui refusent de se coucher ça a été le commencement de l'obscurité.

il reste délicat de statuer sur l'attachement du C-quand à l'ensemble de ce qui précède (le $C$-quand serait alors épitaxique) ou au seul $\mathrm{SN}_{\text {/période/ antécédent. Cependant le lien }}$ sémantique et lexicale entre le $\mathrm{SN}_{\text {/période/ }}$ et le $C$-quand irait dans le sens d'un attachement du C-quand au SN. 
En conclusion nous pouvons donc considérer trois types distincts de couple $\{\mathrm{SN}+C$ quand \} sont le tableau ci-dessous récapitule les propriétés.

Tableau 2. Récapitulatif des propriétés des structures $\{\mathrm{SN}+C-q u a n d\}$.

\begin{tabular}{|c|c|c|c|}
\hline Structures & $\begin{array}{c}\mathbf{S N}_{\text {/animé/ }}+\boldsymbol{C} \text { - } \\
\text { quand }\end{array}$ & $\begin{array}{c}\mathbf{S N}_{\text {/datation/ }}+\boldsymbol{C} \text { - } \\
\text { quand }\end{array}$ & \multicolumn{2}{|c|}{$\mathbf{S N}_{\text {/période/ }}+\boldsymbol{C}$-quand } \\
\hline $\begin{array}{c}\text { Cohésion } \\
\text { syntaxique }\end{array}$ & $\begin{array}{c}\text { Proportionnel à } \\
\text { ça/cela }\end{array}$ & \multicolumn{2}{|c|}{ Proportionnel à (à) ce moment-là } \\
\hline $\begin{array}{c}\text { Proposition } \\
\text { d'interprétation } \\
\text { syntaxique }\end{array}$ & $\begin{array}{c}\text { de la structure : } \\
\text { nexus }\end{array}$ & $\begin{array}{c}\text { du C-quand }: \\
\text { relative appositive }\end{array}$ & $\begin{array}{c}\text { du } \text { C-quand }: \\
\text { parenthèse explicative }\end{array}$ \\
\hline $\begin{array}{c}\text { Lien sémantique } \\
\text { en SN et } \boldsymbol{C} \text {-quand }\end{array}$ & $\begin{array}{c}\text { Lexical : } \\
\text { coréférentialité }\end{array}$ & $\begin{array}{c}\text { Sémantique : } \\
\text { inclusion } \\
\text { temporelle }\end{array}$ & $\begin{array}{c}\text { Sémantique et/ou lexical : } \\
\text { inclusion temporelle (ex.17), } \\
\text { co-actualisation (ex.36), } \\
\text { antonymie (ex.37)... }\end{array}$ \\
\hline
\end{tabular}

\subsection{Structure \{SAdj. + C-quand\}}

Dans cette section sont analysés les cas moins décrits où le $C$-quand semble dans la dépendance d'un adjectif, soit les énoncés comme le suivant :

39. Mais elle n'a pas peur du tout, elle a appris à lancer ses billes d'un bref coup de pouce et son regard sait être insolent quand il faut. (déjà cité 5)

Ici plus encore que pour la structure $\{\mathrm{SN}+C$-quand $\}$ précédente il est délicat de définir l'attachement du $C$-quand, ainsi qu'en attestent les manipulations ci-dessous :

40. Un conducteur prudent quand il le faut, mais rapide quand il le peut. (exemple proposé par A. Berrendonner)

a. extraction de $C$-quand seul : c'est quand il le faut qu'un conducteur est prudent / c'est quand il le peut qu'il est rapide.

a'. extraction de l'ensemble \{SAdj. + C-quand $\}$ : c'est prudent quand il le faut qu'est un conducteur / c'est rapide quand il le peut qu'est un conducteur.

b. proportionnalité du C-quand seul : un conducteur prudent/rapide à ce moment-là/quand? - quand il faut / quand il peut.

b' proportionnalité de l'ensemble $\{$ SAdj. $+C$-quand $\}$ : [c'est] un conducteur ainsi / comment? - prudent quand il faut mais rapide quand il peut.

c. mobilité du $C$-quand seul $^{\mathrm{xii}}$ : quand il le faut c'est un conducteur prudent / quand il le peut c'est un conducteur rapide.

Il semble donc qu'il faille postuler la possibilité d'une double analyse : le C-quandd'un point de vue strictement sémantique - semble bien compléter l'adjectif antécédent dont il restreint l'extension, mais d'un point de vue strictement syntaxique, les deux analyses sont possibles.

Une remarque encore sur la syntaxe interne, cette double analyse parait plus problable lorsque le $C$-quand comprend un verbe modal, et nous semble caduque lorsqu'il n'en contient pas (le $C$-quand serait alors un ajout (circonstant) ordinaire) :

41. Son regard sait être insolent quand il fait beau.

\subsection{Structure $\{$ SAdv. + C-quand $\}$}


On retrouve la même hésitation quand la description syntaxique des structures où le $C$ quand semble dans la dépendance d'un adverbe temporel (désormais SAdv.), soit les énoncés suivants :

42. et maintenant, quand les seigneurs se battent, c'est nous les pauvres qui souffrons... (JLM 12/99)

43. les îlotiers traitent les problèmes immédiats et concrets, et maintenant, quand on va au commissariat, on nous écoute. (JLM 12/99)

44. alors, quand il travaillait aux champs, mon professeur prenait un autre instrument avec lui, une cithare monocorde utilisée dans les chansons de propagande. (JLM 12/99)

45. mais récemment, quand il est allé à Mostar, il est resté plusieurs heures assis dans un café en attendant que passe une tête connue. (JLM 12/99)

Au plan syntaxique, ainsi que pour les structures en $\{\mathrm{SAdj} .+C$-quand $\}$ il est délicat de montrer que l'adverbe et le $C$-quand entretiennent une relation particulière :

46. a. extraction du C-quand seul : C'est quand il travaillait aux champs qu'alors mon professeur prenait un autre instrument avec lui

a'. extraction de l'ensemble $\{\mathrm{SAdv}$. $+C$-quand $\}$ : c'est alors, quand il travaillait au champs, que mon professeur prenait un autre instrument avec lui

b. proportionnalité du $C$-quand seul : alors mon professeur prenait un autre instrument avec lui à ce moment-là / quand? - quand il travaillait aux champs b' proportionnalité de l'ensemble $\{\mathrm{SAdv} .+C$-quand $\}$ : mon professeur prenait un autre instrument avec lui à ce moment-là/quand? - alors quand il travaillait aux champs

c. mobilité du $C$-quand seul : alors mon professeur prenait un autre instrument quand il travaillait aux champs

c' mobilité de la structure $\{\mathrm{SAdv} .+C$-quand $\}$ : mon professeur prenait un autre instrument alors quand il travaillait aux champs

Par conséquent il est difficile encore de statuer sur l'une ou l'autre interprétation. Pourtant, au plan strictement sémantique, il semble bien qu'il faille considérer le lien temporel entre l'adverbe temporel et le $C$-quand, ce rapport semble être équivalent à celui que l'on a pu observer dans les structures en $\left\{\mathrm{SN}_{/ \text {datation } /}+C\right.$-quand $\}$, à savoir une inclusion temporelle à recouvrement partiel du $C$-quand dans le champ de l'adverbe temporel.

Mais parallèlement à ces structures où le $C$-quand est inclus dans l'extension de l'adverbe de temps, il existe des cas où l'ensemble de la structure \{SAdv. + C-quand\} semble jouir d'une relative autonomie énonciative comme dans les exemples ci-dessous :

47. [...] M. Cendré, lui, tape juste ce qu'il faut, pas plus, juste quand il faut. Bo... boum. Tsin, tsin, tsglinng ! C'est la délicatesse française, comme dit maman. (FT : Cavanna F., Les Ritals, 1978)

48. Vous savez, ceux qui sont toujours dans le bon et qui prennent, juste quand il faut, des positions souveraines ; je ne sais pourquoi, ces gens-là - qui sont professionnellement incapables d'erreur - m'ont toujours inspiré une certaine suspicion (FT : Mertens P., Les Éblouissements, 1987)

Dégroupage plus marqué encore par la ponctuation dans (49) et (50) ci-dessous :

49. Punition pour tout le monde. Nous sommes de fameux voyous. Nous finirons aux galères. Les voyous, pour le moment, sont les galériens du français. Surtout quand il faut raconter quelque chose par écrit. (FT : Hélias P. J., Le Cheval d'Orgueil: mémoires d'un Breton du pays bigouden, 1975)

50. - ça y est ! dit La Menace, un zig qui veut de l'essence! Faut que j’y aille! Bon sang, toujours quand il faut pas! (déjà cité 8) 
Dans ces occurrences, on retrouve la contrainte de présence d'un verbe de modalité dans le $C$-quand, mais l'ensemble de la structure $\{\mathrm{SAdv} .+C$-quand $(\subset$ Vmodalité) $\}$ parait de l'ordre de la parenthèse énonciative, apportant un commentaire anaphorique sur le dire antérieur (respectivement sur les termes tape (47), prennent (48) et voyous (49)), ou un commentaire déictique portant sur la situation d'énonciation, comme en (50) que l'on peut ainsi paraphraser : <on me sollicite systématiquement lorsque je ne suis pas disponible $>$.

Il semble donc bien qu'il faille identifier deux types de structure en $\{\mathrm{Sadv} .+C$-quand $\}$ dont le tableau ci-dessous récapitule les propriétés.

Tableau 3. Récapitulatif des propriétés des structures $\{\mathrm{SAdv} .+C$-quand $\}$.

\begin{tabular}{|c|c|c|}
\hline $\begin{array}{l}\text { Structures } \\
\text { Propriétés }\end{array}$ & SAdv./temps/ $+C$-quand & SAdv./temps/ $_{\text {( }}+$-quand $_{(\subset \text { Vmodalité})}$ \\
\hline $\begin{array}{l}\text { Cohésion } \\
\text { syntaxique }\end{array}$ & Ambiguïté analytique & Dégroupage de la structure \\
\hline $\begin{array}{c}\text { Proposition } \\
\text { d'interprétation } \\
\text { syntaxique }\end{array}$ & $\begin{array}{c}\text { analyses pour } C \text {-quand: } \\
\text { - régi ordinaire; } \\
\text { - complément de l'adverbe. }\end{array}$ & $\begin{array}{c}\text { Parenthèse énonciative : } \\
C \text {-quand complément de } \\
\text { l'adverbe. }\end{array}$ \\
\hline $\begin{array}{l}\text { Relation } \\
\text { sémantique }\end{array}$ & $\begin{array}{l}\text { Entre SAdv. et } \boldsymbol{C} \text {-quand } \text { : } \\
\text { inclusion temporelle avec } \\
\text { recouvrement partiel }\end{array}$ & $\begin{array}{c}\text { Entre SAdv. et } \boldsymbol{C} \text {-quand : } \\
\text { inclusion temporelle avec } \\
\text { recouvrement partiel } \\
\text { Entre structure et hôte : } \\
\text { commentaire anaphorique ou } \\
\text { déictique }\end{array}$ \\
\hline
\end{tabular}

\subsection{Structure $\{$ Prép. + C-quand $\}$}

Il s'agit des cas où le $C$-quand semble dans le régime de la préposition (désormais Prép.), soit les exemples suivants :

51. Au début, c'est sympa, on boit des verres. Mais après, quand il faut y aller, tout se complique. (FT : Desplechin M., Sans moi, 1998)

52. qu'ensuite on voudrait tout garder, à cause du souci qu'elles ont donné, du travail de chien que c'est avant de dire « moteur », et pendant la prise, et encore après quand il faut recommencer, l'équipe et les acteurs qui s'échinent et rechignent, les anxieux, les m'as-tu-vu et les je-m'en-foutistes, les emmerdeurs maniaques, la lumière (FT : Garat A.-M., Merle, 1996)

53. Tu as raison. La honte ça ne mène nulle part, crois-moi... La honte que t'as elle te sert à rien. Elle est juste là pour faire plaisir aux braves gens... Après quand $y$ referment leurs volets ou qu'y reviennent du café, y se sentent bien chez eux. Tout rengorgés, y z'enfilent leurs chaussons et se regardent en souriant. (FT : Gavalda A., Ensemble, c'est tout, 2004)

Dans ces exemples l'identification de l'attachement du $C$-quand à la préposition semble moins problématique que dans les structures en $\{\mathrm{SAdj} . / \mathrm{Adv} .+C$-quand $\}$, ainsi qu'en atteste l'inacceptabilité des manipulations (54b,b' et 54c,c') ci-dessous :

54. a. extraction du C-quand seul : C'est quand il faut $y$ aller qu'après tout se complique (déjà cité 51)

a'. extraction de l'ensemble \{Prép. $+C$-quand : c'est après, quand il faut $y$ aller que tout se complique

b. proportionnalité du $C$-quand seul : ? après tout se complique à ce moment-là / quand? - quand il faut y aller 
b' proportionnalité de l'ensemble \{Prép. $+C$-quand $\}$ ? tout se complique à ce moment-là / quand? - après quand il faut y aller

c. mobilité du $C$-quand seul : ? après tout se complique quand il faut y aller c' mobilité de la structure \{Prép. $+C$-quand $\}$ : tout se complique après quand il faut y aller.

D'un point de vue sémantique on peut considérer que le rapport temporel entre la préposition et le $C$-quand est bien un rapport d'inclusion, le $C$-quand apparaissant alors dans le régime syntaxique et l'extension sémantique de la préposition, ce que montre la délicate suppression du C-quand:

55. \# Au début, c'est sympa, on boit des verres. Mais après $\varnothing$ tout se complique.

56. \# qu'ensuite on voudrait tout garder, à cause du souci qu'elles ont donné, du travail de chien que c'est avant de dire « moteur », et pendant la prise, et encore après $\varnothing$ l'équipe et les acteurs qui s'échinent et rechignent [...]

57 . \# [...] La honte que t'as elle te sert à rien. Elle est juste là pour faire plaisir aux braves gens... Après $\varnothing$ y se sentent bien chez eux.

L'absence de $C$-quand force à chercher une information, facilement inférable du contexte antérieur, ce que l'on pourrait ainsi lexicaliser :

58. Au début, c'est sympa, on [boit des verres] $]_{\mathrm{i}}$. Mais après avoir bu des verres tout se complique.

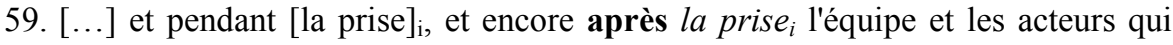
s'échinent et rechignent $[\ldots]$

60. [...] La honte que t'as elle te sert à rien. Elle est juste là pour [faire plaisir aux braves gens $]_{i} \ldots$ Après avoir fait plaisir aux braves gens s $_{i} \mathrm{y}$ se sentent bien chez eux.

L'absence de $C$-quand modifie donc la structuration syntaxique et impose l'ellipse. Le $C$-quand dans ce contexte semble donc assurer la complétude syntaxique et sémantique de la préposition à valeur temporelle.

\section{Statut de quand}

Sans prétendre trancher la question du statut de quand d'un point de vue global, nous tenterons de considérer son statut pour les structures étudiées.

Dans l'ensemble des structures, quand semble proportionnel à au moment où :

61. C'était celle de Mathilde quand/au moment où elle était petite... (déjà cité 1)

62. L'actuelle vague de violences s'est ouverte fin novembre quand/au moment où en l'espace d'une seule journée plus de 40 personnes ont été tuées (déjà cité 3)

63. Les heures de la contestation, quand/au moment où Beaubourg était moqué pour ses allures d'usine à gaz, semblent bien lointaine. (déjà cité 4)

64. Mais elle n'a pas peur du tout, elle a appris à lancer ses billes d'un bref coup de pouce et son regard sait être insolent quand/au moment où il faut. (déjà cité 5)

65. " et maintenant, quand/au moment où les seigneurs se battent, c'est nous les pauvres qui souffrons... » (déjà cité 6)

66. Mais après, quand/au moment où il faut y aller, tout se complique. (déjà cité 51)

Cette proportionnalité entre quand et au moment où invite à considérer le marqueur comme une proforme relative à antécédent incorporé. 


\section{Conclusion}

Si d'un point de vue syntaxique il est parfois délicat de statuer de façon certaine sur l'attachement ou l'inclusion du $C$-quand dans un hôte non verbal, il n'en reste pas moins que d'un point de vue sémantique, il semble bien que les $C$-quand examinés viennent restreindre ou expliciter le constituant hôte, s'apparentant de fait au fonctionnement des relatives appositives.

\section{Références bibliographiques}

Béguelin, M.-J., (2004), « Coalescences et dégroupages syntaxiques : réflexions sur les conditions pragmatiques et sémantiques des grammaticalisations ». In A. Auchlin et al. (éds), Structures et discours. Mélanges offerts à Eddy Roulet. Québec : Éditions Nota Bene, 2004, 139-150, en ligne.

Benzitoun, C., (2006), Description morphosyntaxique du mot «quand» en français contemporain, Thèse de doctorat de l'université de Aix-Marseille I.

Benzitoun, C., (2008), «Qui est quand ? Essai d'analyse catégorielle », Langue Française 2|2008 $\mathrm{n}^{\circ} 158,129-143$.

Benzitoun, C. et Saez, F. (2016), notice sur «Les constituants en quand en français », in Encyclopédie Grammaticale du Français, http://encyclogram.fr.

Berrendonner, A. et Reichler-Béguelin M.-J., (1989), «Décalages: les niveaux de l'analyse linguistique », Langue française $81 / 1$, Structuration de textes : connecteurs et démarcations graphiques, 99-125, en ligne : http://www.persee.fr

Berrendonner, A., (1983), "Connecteurs pragmatiques et anaphore», Cahiers de linguistique française 5, 215-246, en ligne : http://clf.unige.ch/num.php?numero=5

Berrendonner, A., (1990), «Pour une macro-syntaxe », Travaux de linguistique 21, 25-36.

Blanche-Benveniste, C., Delofeu, J., Stefanini, J. \& Eynde, K. van den, (1984), Pronom et syntaxe. L'approche pronominale et son application au français, Paris: SELAF, pp. 245.

Borillo, A., (1988), «Quelques remarques sur quand connecteur temporel », Langue française 77, 7191.

Chétrit, J., (1976), Syntaxe de la phrase complexe a subordonnée temporelle, étude descriptive, Paris : Klincksieck

Eynde, K. van den \& Blanche-Benveniste, C., (1978), «Syntaxe et mécanismes descriptifs : présentation de l'approche pronominale », Cahiers de Lexicologie 32, 3-27.

Maurel, J.-P., (1992), «Subordination inverse et neutralisation du relatif. », Travaux de linguistique du CerLICO 5, $72-88$

Muller, C., (1996), La subordination en français, Paris : Armand Colin

Riegel, M., Pellat J.-C., Rioul, R., (1994, rev. 2009), Grammaire méthodique du français, Paris: P.U.F.

Saez, F., (2011), La scalarité de l'intégration syntaxique: étude syntaxique, sémantique et pragmatique de la proposition en quand, Thèse de doctorat de l'université de Toulouse 2-Le Mirail.

Sandfeld, K., (1965), Syntaxe du français contemporain, les propositions subordonnées, 2e ed., Genève (Suisse) : Droz S.A.

Smessaert, H., Cornillie, B., Djivar, D., \& Eynde, K. van den, (2005), "Degrees of clause integration from endotactic to exotactic subordination", Linguistics 43, 471-529. 


\footnotetext{
${ }^{\mathrm{i}}$ Le terme de constituant est ici préféré à celui de proposition car il permet d'éviter d'anticiper sur la nature de la relation entre le constituant introduit et son terme hôte.

ii Toute prudence gardée ici par rapport à cette notion de dépendance que nous préciserons dans l'article.

iii Nous utilisons le terme neutre de élément pour ne pas enclore l'hôte du C-quand dans une seule catégorie.

${ }^{\text {iv }}$ Dans les exemples, nous soulignons.

${ }^{\mathrm{v}}$ Voir entre autres Benzitoun et Saez (2016).

${ }^{v i}$ Voir également Saez (2011: 261 et suiv.) pour plus de précision sur les relations sémanticosyntaxique entre l'hôte $\mathrm{SN}_{\text {/temps/ }}$ et le $C$-quand.

${ }^{v i i}$ Le terme de constituants temporels est entendu dans un sens très large, suivant Béguelin (2004), incluant dans cette catégorie l'aspect du verbe recteur.

viii Le symbole « T $\subset$ » signifie « inclut temporellement ».

${ }^{\mathrm{ix}}$ Saez $(2011: 261$ et suiv. $)$.

${ }^{\mathrm{x}}$ Toute prudence gardée vis-à-vis de la paraphrase.

${ }^{x i}$ Dans le sens de Borillo (1988): une situation (C-quand) intervient dans le cadre d'une situation déjà en cours (hôte du $C$-quand).

${ }^{x i i}$ En raison de la construction attributive, il est délicat d'antéposer l'ensemble $\{\operatorname{Sadj}+C$-quand $\}$
} 